Published in final edited form as:

Nat Microbiol. 2019 June ; 4(6): 996-1005. doi:10.1038/s41564-019-0383-z.

\title{
Genome mining identifies cepacin as a plant-protective metabolite of the biopesticidal bacterium Burkholderia ambifaria
}

\author{
Alex J. Mullins ${ }^{1}$, James. A. H. Murray ${ }^{1}$, Matthew J. Bull ${ }^{1}$, Matthew Jenner ${ }^{2}$, Cerith Jones ${ }^{1,6}$, \\ Gordon Webster ${ }^{1}$, Angharad E. Green ${ }^{3}$, Daniel R. Neill ${ }^{3}$, Thomas R. Connor ${ }^{1}$, Julian \\ Parkhill $^{4}$, Gregory L. Challis ${ }^{2,5}$, and Eshwar Mahenthiralingam ${ }^{1}$ \\ ${ }^{1}$ Microbiomes, Microbes and Informatics Group, Organisms and Environment Division, School of \\ Biosciences, Cardiff University, Cardiff CF10 3AX, United Kingdom \\ ${ }^{2}$ Department of Chemistry and Warwick Integrative Synthetic Biology Centre, University of \\ Warwick, Coventry CV4 7AL, United Kingdom \\ ${ }^{3}$ Institute of Infection and Global Health, University of Liverpool, Liverpool L69 7BE, United \\ Kingdom \\ ${ }^{4}$ Wellcome Sanger Institute, Wellcome Genome Campus, Hinxton, Cambridge CB10 1SA, United \\ Kingdom
}

\begin{abstract}
Users may view, print, copy, and download text and data-mine the content in such documents, for the purposes of academic research, subject always to the full Conditions of use:http://www.nature.com/authors/editorial_policies/license.html\#terms

Corresponding Author: Submission and handling: Prof. Eshwar Mahenthiralingam, Microbiomes, Microbes and Informatics Group, Organisms and Environment Division, School of Biosciences, Cardiff University, Sir Martin Evans Building, Museum Avenue, Cardiff CF10 3AX, United Kingdom; Tel. +44 (0)29 20875875; Fax. +44 (0)29 20874305; MahenthiralingamE@ cardiff.ac.uk. Joint correspondence: Alex J. Mullins, Microbiomes, Microbes and Informatics Group, Organisms and Environment Division, School of Biosciences, Cardiff University, Sir Martin Evans Building, Museum Avenue, Cardiff CF10 3AX, United Kingdom; Tel. +44 (0)29 20874648 MullinsA@cardiff.ac.uk.

${ }^{6}$ Current address: Faculty of Computing, Engineering and Science, University of South Wales, Pontypridd, CF37 1DL, United Kingdom

Data Availability

Sequence data that support the genomic findings of this study have been deposited in the European Nucleotide Archive with the accession/bioproject codes listed in Supplementary Table 1. The data that support the antimicrobial production, $P$. sativum and $G$. mellonella survival, and murine infection model findings of this study are available from the corresponding authors upon request. Bacterial strains and constructs will be made available upon written request to the corresponding authors and after signing a Material Transfer Agreement. We are restricted in re-distributing certain bacterial strains such as those from recognised culture collections, but such requests will be re-directed to the appropriate source.
\end{abstract}

Code Availability

The publicly available software and codes used for genome sequence determination, phylogenomics, mass spectrometry and general statistical analysis are described in the appropriate Method sections.

Author contributions:

The initial study to characterise the genomes of $B$. ambifaria as a biopesticide was conceived by EM, with additional aspects of the study design added by AM, GC and JM. AM performed all aspects of the study with the exception of the LC-MS profiling, and was assisted by specific contributions from the following: datasets and input for genome sequencing and mining, EM, GC, JP and TC; genome assembly, phylogenomics, cluster mining and de-replication, MB; LuxR mining, EM; generation of a cepacin insertional mutant and antimicrobial activity screening, $\mathrm{CJ}$; extraction, identification and fractionation of Burkholderia metabolites by HPLC, and enacyloxin MIC analysis, GW; LC-MS identification and confirmation of $B$. ambifaria antimicrobial metabolites, MJ and GC; biocontrol modelling EM, GW, and JM; evaluation and analysis of plant models, JM; Galleria virulence assays, GW and CJ; and murine infection modelling and analysis, AG and DN. AM and EM developed the first draft of the manuscript and all authors read and contributed towards finalisation of the study.

Competing interests:

The authors do not have any competing interest to declare.

Eshwar Mahenthiralingam (ORCID: 0000-0001-9014-3790)

Alex J. Mullins (ORCID: 0000-0001-5804-9008) 
${ }^{5}$ Department of Biochemistry and Molecular Biology, Biomedicine Discovery Institute, Monash University, Clayton, VIC 3800, Australia

\section{Abstract}

Beneficial microorganisms are widely used in agriculture for control of plant pathogens but a lack of efficacy and safety information has limited the exploitation of multiple promising biopesticides. We applied phylogeny-led genome mining, metabolite analyses and biological control assays to define the efficacy of Burkholderia ambifaria, a naturally beneficial bacterium with proven biocontrol properties, but potential pathogenic risk. A panel of 64 B. ambifaria strains demonstrated significant antimicrobial activity against priority plant pathogens. Genome sequencing, specialized metabolite biosynthetic gene cluster mining and metabolite analysis revealed an armoury of known and unknown pathways within $B$. ambifaria. The biosynthetic gene cluster responsible for the production of the metabolite, cepacin, was identified and directly shown to mediate protection of germinating crops against Pythium damping-off disease. B. ambifaria maintained biopesticidal protection and overall fitness in soil after deletion of its third replicon, a non-essential plasmid associated with virulence in B. cepacia complex bacteria. Removal of the third replicon reduced $B$. ambifaria persistence in a murine respiratory infection model. Here we show that by using interdisciplinary phylogenomic, metabolomic and functional approaches, the mode of action of natural biological control agents related to pathogens can be systematically established to facilitate their future exploitation.

\section{Keywords}

Burkholderia; biopesticides; phylogenomics; genome mining; specialized metabolites

Numerous bacterial and fungal species have been recognised for their biological control abilities and plant growth-enhancing properties. Pesticides conventionally used in agriculture are under increasing scrutiny regarding their bioaccumulation and toxicity, which includes their fatal impact on pollinator species. Concern over chemical pesticides has reinvigorated research into biological control agents and their secreted bioactive compounds as viable natural alternatives for agriculture. One feature common to most biopesticidal species is their ability to secrete antimicrobial compounds into the environment and inhibit pathogenic microbes from causing crop disease. Bacteria within the genus Burkholderia are particularly diverse in their specialized metabolism and have a documented ability to produce a range of potent anti-bacterial, anti-nematodal and anti-fungal compounds1,2. They have demonstrated excellent promise as biological control agents with multiple strains used commercially as biopesticides until 1999. In common with other biological control genera such as Bacillus, Pseudomonas and Stenotrophomonas, certain Burkholderia species may also cause human, animal and plant infections. Therefore, in 1999 the US Environmental Protection Agency (EPA) placed a moratorium on new registrations of Burkholderia biopesticides unless such agents were defined as safe in terms of their risk of opportunistic infection2.

Multiple species within the Burkholderia cepacia complex group were characterised or used as biological control agents2. They are highly active in their specialized metabolism, for 
example, producing antifungal compounds including pyrrolnitrin, occidiofungin, cepafungin and burkholdines; antibacterial bactobolins and enacyloxin IIa; and broader spectrum agents such as the cepacins3,4. Outside of the B. cepacia complex, other Burkholderia species also produce a range of antagonistic compounds. The bactobolins are also produced by Burkholderia thailandensis and Burkholderia pseudomallei and exhibit potent activity against Gram-positive bacteria, including methicillin-resistant Staphylococcus aureus5. Burkholderia gladioli also produces multiple antimicrobials including the anti-mycobacterial macrolide gladiolin6, its isomer lagriene7, the cytotoxic azapteridine toxoflavin8, and the polyyne caryoynencin9. There is no consensus on the distribution or co-occurrence of antimicrobial specialized metabolite encoding gene clusters in biopesticidal B. ambifaria, nor a holistic understanding of strain bioactivity and antimicrobial compound efficacy against priority bacterial10,11, fungal and oomycetal plant pathogens2. Biopesticidal activities have been descriptively characterised for individual producer strains against a panel of target organisms, or multiple producer strains against relatively few target organisms2. Previous studies have analysed the overall antagonistic properties of $B$. ambifaria, but none have examined the role of specific antimicrobial compounds in mediating biocontrol in natural soil microcosm models.

To establish a biotechnological platform for biopesticidal use of $B$. ambifaria that considers its efficacy and safety, we systematically defined the genomic basis and functional efficacy of antimicrobial metabolites in 64 strains including 8 previously characterised biocontrol strains (Supplementary Table 1). The strain collection examined included 58 environmental isolates recovered from multiple sources (soil, the maize, pea and grass rhizosphere, and leaves) and 6 strains isolated from the sputum of people with cystic fibrosis (CF). Collectively, it also represented strains recovered from various geographic origins (USA, Australia and Italy; Supplementary Table 1). The B. ambifaria core and accessory genome was revealed, and gene cluster network analyses were combined with antimicrobial activity assays to rationally understand the biopesticidal activity against crop pathogens. The role of individual antimicrobial metabolites in mediating crop protection was investigated using biosynthetic pathway mutants in non-sterile soil biocontrol models. Since curing of the $B$. cepacia complex third genomic replicon (c3) is possible in these multireplicon bacteria12, a B. ambifaria $\mathrm{c} 3$ mutant was constructed and shown to have reduced virulence in a murine respiratory infection model, yet retained its plant-protective properties. This work provides a foundation for developing targeted biological control agents and effective biocontrol products for reducing agricultural crop losses from bacterial, fungal and oomycete pathogens.

\section{Results}

\section{B. ambifaria genomics and in silico definition of specialized metabolite biosynthetic gene clusters}

To understand the genome-encoded potential of $B$. ambifaria as a biopesticide, phylogenomic and pan-genomic analyses were applied (see Methods, Supplementary Figure 1, Supplementary Table 2 and Supplementary Notes). The three replicon genomic structure was present in 63 of the B. ambifaria strains analysed, while strain BCC1105 naturally 
lacked the third replicon. Contigs were scaffolded to one of three reference genomes to assemble complete genomes. The assembled genome sizes varied across the 64 strains, from $6.13 \mathrm{Mbp}$ (BCC1105) to $8.03 \mathrm{Mbp}$ (BCC1248), with a mean of 7.34 Mbp (Supplementary Table 2). Assembled replicons c1, c2 and c3 possessed a mean of 3.47 Mbp, 2.74 Mbp and $1.15 \mathrm{Mbp}$, respectively. Replicon c3 possessed the greatest variation in sequence capacity, whereas replicons c2 and c1 displayed greater consistency in size (Supplementary Table 2). A large B. ambifaria pan-genome was identified (22,376 distinct genes) of which 3784 genes comprised the core genome. The pan-genome represented a collection of genes approximately 3.4-fold greater than the mean $B$. ambifaria genome (6,546 genes). A large proportion of the accessory genome, $78.1 \%$ (14,582 genes), was shared by less than $15 \%$ of the B. ambifaria strains. Exclusion of the strain which lacked the third replicon, BCC1105, from the core genome analysis resulted in the $B$. ambifaria core genome increasing from 3784 to 4166 genes. Three major clades were identified in the $B$. ambifaria 3784 core-gene phylogeny (Figure 1A), and this established the evolutionary framework onto which the antimicrobial properties of each strain were overlaid using in silico and bioactivity approaches. In silico analyses of the 64 B. ambifaria genomes (see Methods, Supplementary Table 3 and Supplementary Notes) detected 1272 specialized metabolite biosynthetic gene clusters (BGCs), that were de-replicated into 38 distinct BGCs after Kmer-matching and gene topology comparisons (Figure 2; Supplementary Table 3). Network analysis was used to graphically summarise multiple attributes of the $B$. ambifaria BGCs including their biosynthetic diversity, strain distribution, and core or accessory nature within the species (Figure 2).

Of the 38 distinct BGCs, 13 were previously characterised, and seven known to encode compounds with antimicrobial activity (Supplementary Table 4). Pyrrolnitrin13 was the only BGC for an antimicrobial metabolite found in all 64 B. ambifaria strains, whereas the BGC for the anti-Gram-negative metabolite enacyloxin IIa3 was the least common known antimicrobial BGC (Figure 2). Pyrrolnitrin and phenazine BGCs were encoded on replicon c2 and the remaining antagonistic compounds were encoded by BGCs on replicon c3. No known antimicrobial BGCs were identified on replicon c1. Barring a few exceptions, multiple antimicrobial encoding BGCs were associated with distinct clades within the $B$. ambifaria core-gene phylogeny (Figure 1). Six of the seven clade $1 \mathrm{~b}$ strains encoded the pathway responsible for enacyloxin IIa biosynthesis3. The more widely distributed burkholdine14 BGC was absent from all members of clade 2 and strain BCC1105, but all other strains possessed the anti-fungal biosynthetic locus. Bactobolin5 BGCs were concentrated in clade 1 and less frequently encountered in clade 2 and 3 strains. Two strains, BCC1105 and BCC1224, only encoded the core anti-fungal metabolite pyrrolnitrin, and lacked any additional antimicrobial BGCs (Figure 1B). No single strain encoded all 7 previously known antimicrobial BGCs, however, approximately $59 \%$ of strains encoded four or more BGCs reflecting the known antimicrobial properties of $B$. ambifaria (Figure 1B).

The silent nature of certain antimicrobial BGCs which are not expressed in standard laboratory cultures, including those in Burkholderia, is well established3,4. We therefore correlated in vitro metabolite production with BGC distribution. Ten B. ambifaria strains representing the seven characterised biocontrol strains and three additional strains from the broader species phylogeny (Figure 1A) were screened for metabolite production on agar 
growth media BSM-G3,15. Six known antimicrobial metabolites were detected by LC-MS (Supplementary Figure 2 and 3), five of which could be directly correlated to the presence of predicted BGCs (Table 1). Under these screening conditions the majority of BGCs (22 of 25 ) were biosynthetically active and produced the corresponding metabolite; individual strains encoding pyrrolnitrin, burkholdines and hydroxyquinolines BGCs were exceptions to this trend (Table 1). A sixth known metabolite, cepacin A16, was also detected in $B$. ambifaria J82 (BCC0191) by analytical analyses (see Methods) and subsequently correlated to a BGC (not recognised by antiSMASH v3) 17 identified by searching for quorum sensing (QS) regulated BGCs (see below).

\section{Mapping direct antimicrobial activity against plant and animal pathogens}

Having established the presence of BGCs (Figures $1 \mathrm{~b}$ and 2 ) and corresponding metabolites (Table 1), antagonism activity of the $64 \mathrm{~B}$. ambifaria strains against priority plant10 and human pathogens (Supplementary Table 5) was evaluated as described3. The in vitro bioactivity was aligned against the core-gene phylogeny to map antagonism across $B$. ambifaria as a species (Figure 1c). A total of six strains lacked observable antimicrobial activity (Figure 1c). Clade 1a, $1 \mathrm{~b}$ and $1 \mathrm{c}$ strains exhibited substantial bioactivity against Gram-negative pathogens, while only two strains outside these clades exhibited similar activity (Figure 1c). Clade 1b strains exhibited additional strong antagonistic activity towards Betaproteobacteria, Burkholderia multivorans and Alphaproteobacteria, Rhizobium radiobacter (Figure 1c), an activity not observed in other anti-Gram-negative B. ambifaria strains. The extended antimicrobial antagonism of clade $1 \mathrm{~b} B$. ambifaria correlated to the presence of the trans-AT PKS BGC for enacyloxin IIa (Figure 1b); all screened Gramnegative pathogens were susceptible to purified enacyloxin IIa with MICs ranging from 3.2 to $50 \mu \mathrm{g} / \mathrm{ml}$ (Supplementary Table 6). The additional anti-Gram-negative activity correlated to the presence of the hybrid NRPS-PKS encoding BGC for bactobolin (Figure 1b). Antifungal (against Candida albicans, Fusarium solani and Alternaria alternata) and anti-Grampositive (against Staphylococcus aureus, Enterococcus faecalis and Bacillus subtilis) activity was more widespread than anti-Gram-negative activity in B. ambifaria, with $82 \%$ and $69 \%$ of tested strains (62 of 64) exhibiting these activities, respectively (Figure 1). Clade $2 B$. ambifaria strains exhibited the least antimicrobial activity (5 of 9 lacking any in vitro observable activity) despite encoding BGCs for pyrrolnitrin, hydroxyquinolines, and cepacin (Figure 1b).

\section{A search for QS-regulated BGCs reveals the biosynthetic locus for the potent anti- oomycetal cepacin}

Multiple specialized metabolite BGCs are QS controlled3,4 and manipulation of this regulatory system has also been harnessed for Burkholderia metabolite discovery18. A search of $\operatorname{lux} R$ homologues within the $B$. ambifaria genomes was performed (see Supplementary Notes) and downstream of an uncharacterised LuxRI system (encoded by 22 of 64 strains) (Figure 3) was a conspicuous BGC encoding fatty acid desaturases, a betaketoacyl synthase and an acyl carrier protein (Figure 4a). Insertional mutagenesis of a fatty acyl-adenosine monophosphate (AMP) ligase-encoding gene, $c c n J$, within this BCG was carried out in six B. ambifaria strain backgrounds (BCC0191, BCC1252, BCC1241, BCC0477, BCC1259 and BCC1218; Figure 1). The resulting mutants lacked anti-Gram- 
positive activity and the weak anti-Gram-negative activity, and showed considerably diminished growth inhibition of the oomycete Pythium ultimum (Figure $4 \mathrm{~b}$ and Supplementary Figure 4). High resolution mass spectrometry of metabolite extracts from strain BCC0191 identified ions with $\mathrm{m} / \mathrm{z}=271.0964$, corresponding to a predicted molecular formula of $\mathrm{C}_{16} \mathrm{H}_{14} \mathrm{O}_{4}$ consistent with cepacin $\mathrm{A}$, a historically described Burkholderia polyyne16 of un-defined biosynthetic origin. Direct comparison with extracts from the originally reported cepacin A and cepacin B producer strain, "B. cepacia" ATCC 3935616 (taxonomically reclassified as a Burkholderia diffusa strain), confirmed that $B$. ambifaria BCC0191 produces cepacin A (Supplementary Figure 5). Cepacin A was absent in the B. ambifaria BCC0191::ccnJ cepacin insertional mutant (Figure 4c), confirming that the uncharacterised LuxRI-associated BGC was responsible for the biosynthesis of this known Burkholderia metabolite. The cepacin A BGC is located on the second replicon of $22 \mathrm{~B}$. ambifaria strains, with $100 \%$ and $56 \%$ presence in clade 3 and clade 2 strains, respectively (Figure 1b).

\section{Cepacin A is a key mediator of B. ambifaria biocontrol of Pythium ultimum damping-off disease}

B. ambifaria has been observed to inhibit $P$. ultimum and application to prevent crop damping-off diseases was a key trait in its historical biopesticide use2. However, the metabolites and/or BGCs which drive Burkholderia crop protection against Pythiummediated damping-off have not been defined in a relevant biopesticide model, such as bacterial seed coating and planting in pathogen infested soil2. The cepacin-producer $B$. ambifaria BCC0191 exhibited strong biopesticidal activity when introduced as a $P$. sativum (pea) seed-coat to a P. ultimum biocontrol model in non-sterile soil (Figure 5a). Disruption of the cepacin BGC and application of the BCC0191 cepacin mutant as a seed coat reduced pea plant survival rates by more than $60 \%$ dependent on $B$. ambifaria seed coat inoculum level $\left(10^{5}, 10^{6}\right.$ and $10^{7} \mathrm{cfu} / \mathrm{seed}$; Figure $\left.5 \mathrm{a}\right)$. No biological control was observed when $10^{5}$ cfu/seed of BCC0191 cepacin mutant was applied ( $<10 \%$ survival), compared to $>50 \%$ protection mediated by the wild type at this level (Figure 5a).

A unique feature of the $B$. cepacia complex multi-replicon genome is that the third replicon is not essential and $\mathrm{c} 3$ deletion mutants lose virulence and antifungal phenotypes 12 . The cepacin BGC is located on the second $\mathrm{c} 2$ replicon of $B$. ambifaria and its biosynthesis was maintained when a third replicon deletion mutant, BCC0191 $\Delta \mathrm{c} 3$, was constructed. Despite the loss of $>1 \mathrm{Mb}$ of DNA, the BCC0191 $\Delta \mathrm{c} 3$ derivative remained competitive and biopesticidal in the Pythium-infested soil microbial community, protecting peas from damping-off at a rate marginally below that of the wild type (Figure 5b; the difference was not significant for a given inoculation size). The phenotypes of $B$. ambifaria BCC0191, its cepacin-deficient derivative (::ccnJ), c3 knockout mutant $(\Delta \mathrm{c} 3)$, and combined cepacin-c3 mutation $(: \because c c n J \Delta c 3)$ were tested further to understand the wider effect of these mutations on strain fitness (see Supplementary Notes). Antimicrobial activity against Gram-positive bacteria and Pythium was lost in the cepacin deficient mutant (Supplementary Figure 6). The BCC0191 c3 mutant lost antifungal activity but had a 2-fold increase in cepacin production enhancing its anti-Gram-positive antagonism (Supplementary Figure 6). Rhizocompetence was similar for the BCC0191 WT and BCC0191::ccnJ, but the third replicon deletion mutant 
colonised the pea rhizosphere at a significantly lower rate of $8.5 \times 10^{6} \mathrm{cfu} / \mathrm{g}$ root $(\mathrm{p}=0.027$; Supplementary Table 7).

A lack of understanding of safety and human pathogenicity were key reasons the US EPA placed a moratorium on B. cepacia complex biopesticides2. Since the BCC0191 $\Delta \mathrm{c} 3$ mutant had retained its biopesticidal ability (Figure $5 b$ ), yet loss of this replicon is associated with reduced virulence in multiple infection models 12 , we assessed the pathogenicity of $B$. ambifaria BCC0191 and its c3 deletion mutant. In the Galleria mellonella wax-moth larvae model12, the deletion of the third replicon did not attenuate the virulence (Supplementary Figure 7a), showing that genes encoding significant insecticidal pathogenicity were not encoded on $\mathrm{c} 3$ in B. ambifaria strain BCC0191. In contrast, using a murine respiratory infection model relevant to chronic cystic fibrosis lung infections19,20, the persistence of $B$. ambifaria BCC0191 was low and loss of the third replicon BCC0191 $\Delta \mathrm{c} 3$ further reduced persistence in the lung (Supplementary Figure $7 \mathrm{~b}$ and Supplementary Figure 7c). At an infective dose of $2 \times 10^{6}$ bacteria, the BCC0191 wild type persisted in the nasopharynx for the duration of the 5-day experiment but was cleared from lungs of 4 out of 6 mice by day 5 . In contrast, the $\mathrm{c} 3$ mutant was rapidly cleared from both nasopharynx and lungs of mice (Supplementary Figure 8). Low numbers of the parental BCC0191 strain ( $<50$ colonies) were detected in the lungs of mice after 5 days of infection, but BCC0191 $\Delta \mathrm{c} 3$ was cleared within 48 hours. B. ambifaria (wild-type or c3 mutant) was not detected within the spleens of infected mice and no visible disease signs were observed throughout. Genotyping by PCR demonstrated that the low numbers of colonies recovered from the mouse infection model were either the administered B. ambifaria BCC0191 or BCC0191 $\Delta \mathrm{c} 3$, respectively (Supplementary Figure 8).

\section{Discussion}

Harnessing the potential of naturally biopesticidal bacteria is an important consideration if we are to keep pace with agricultural intensification and global food security. With increasing regulatory and environmental scrutiny of pesticides, the properties of natural agents will also have to be systematically defined before widespread use. Our in-depth genomic analysis of the intra-species diversity of $B$. ambifaria as a biopesticide and direct linkage of its specific metabolite, cepacin, to antagonism of Pythium and prevention of crop damping-off disease, sets a precedent on the mode of action of Burkholderia biopesticides. We have developed a holistic understanding of biopesticidal B. ambifaria, determining their pan-genomic content, extensive library of antimicrobial BGCs, efficacy in targeting key plant pathogens with specific antimicrobial metabolites, and defining the population biology of historically applied B. ambifaria biopesticides (see Supplementary Discussion). We have shown that biological control of damping-off disease in a relevant soil model is critically mediated by cepacin A, encoded on the second replicon of $B$. ambifaria. Since effective biological control of Pythium also occurs in the absence of the third replicon, which has been characterised as a Burkholderia virulence plasmid12, we have highlighted this as an attenuation strategy for developing potentially safe biopesticide strains which retain biotechnological efficacy. 


\section{Discovery of cepacin biosynthetic gene cluster}

Mining and phylogenetically clustering the LuxR protein sequences from 64 B. ambifaria genomes revealed multiple solo and $l u x I$-associated $l u x R$ genes, and these were linked with both known and uncharacterised specialized metabolite BGCs. In addition to the $B$. ambifaria encoded enacyloxin3 and bactobolin5 BGCs, LuxR regulation of other specialised metabolite BGCs has been further described within and outside the genus. Burkholderia thailandensis synthesises the quorum sensing regulated cytotoxic compound malleilactone21, and pyocyanin production in Pseudomonas aeruginosa is controlled by a hierarchical QS network22. This approach was initially intended to understand the role of quorum sensing regulation in $B$. ambifaria biopesticidal specialized metabolism, but serendipitously led to the identification of the cepacin A BGC.

Cepacins A and B were initially described as metabolites of Burkholderia cepacia, formally Pseudomonas cepacia16,23, with the original producer strain now classified as B. diffusa24. Both polyyne metabolites displayed strong anti-Staphylococci activity, while cepacin A showed weak anti-Gram-negative activity 16. Cluster K, a gene cluster with 76.9\% homologous nucleotide similarity spanning $12.9 \mathrm{kbp}$ (in addition to $8.4 \mathrm{kbp}$ of nonhomologous regions) to the B. ambifaria cepacin A BGC was identified in Collimonas fungivorans Ter331 using nucleotide BLAST (Supplementary Figure 9). The $C$. fungivorans cluster $\mathrm{K}$ has been linked to the biosynthesis of the anti-fungal polyyne collimomycin25 whose BGC organisation (Supplementary Figure 9) and chemical formula $\left(\mathrm{C}_{16} \mathrm{H}_{18} \mathrm{O}_{4}\right)$ resemble cepacin A $\left(\mathrm{C}_{16} \mathrm{H}_{14} \mathrm{O}_{4}\right)$. Recent characterisation of this $C$. fungivorans strain showed it produces a range of polyynes, collectively designated the collimonins 26 , with collimonin A showing the most structural similarity to cepacin A. Several key differences in gene content were identified between the cepacin and collimonin BGCs in the regions flanking the core biosynthetic genes (Supplementary Figure 9). C. fungivorans cluster K contains genes encoding four additional hypothetical proteins, a major-facilitator superfamily transporter and fatty acid desaturase; whereas the B. ambifaria cepacin BGC encoded one extra hypothetical protein. An unusual feature of the cepacin and collimonin BGC variants is the substitution of the associated regulatory genes that comprise a QSassociated luxRI in Burkholderia and a lysR regulator in Collimonas (Supplementary Figure 9). Similar proteins to those identified in the cepacin BGC are listed in Supplementary Table 8.

\section{Cepacin A is a major component of $B$. ambifaria biological control}

We have demonstrated the importance of cepacin A in the context of biological control of the major crop family Fabaceae (Pisum sativum). Disruption of the cepacin BGC in $B$. ambifaria significantly reduced plant survival beyond germination and emergence compared to the wild-type (Figure 5a; $\mathrm{p}<0.05$ ). The contribution of specific metabolites to biocontrol has been studied extensively in Pseudomonas 27 relative to other characterised biocontrol genera. The anti-fungal properties of pyrrolnitrin and 2,4-diacetylphloroglucinol have been evidenced as important metabolites in the biological control of several fungal pathogens, on a diversity of crops, in a range of Pseudomonas species and strain backgrounds27. Other studies have highlighted the in vitro antimicrobial activity, and presence of the corresponding specialized metabolite BGCs or protective effects in field trials in Bacillus 28 
and Streptomyces29, but fail to define the impact of distinct metabolites in a biocontrol system. This study establishes the role of cepacin A as the major bioactive component of the $B$. ambifaria armoury in the biocontrol of damping-off disease by $P$. ultimum in a relevant non-sterile soil model. The reduced protection against $P$. ultimum of the cepacin A-deficient mutant compared to the wild-type $B$. ambifaria also indirectly confirms the expression of the cepacin A BGC in planta (Figure 5b).

There has been considerable discussion on whether Burkholderia species known to cause opportunistic infections can be safely exploited for environmental benefit30. Multiple species in the recently defined Paraburkholderia genus have not been associated with infection, are generally environmental, and mediate plant-beneficial interactions 30 . Transfer of biopesticidal properties such as the B. ambifaria cepacin BGC to Paraburkholderia species is a potential route to future safe usage. Attenuation of pathogenicity in biopesticidal strains is an alternative means to facilitate their biotechnological exploitation. Third replicon deletion in B. ambifaria BCC0191 led to loss of persistence in the murine lung infection model (Supplementary Figure $7 \mathrm{~b}$ and $7 \mathrm{c}$ ), and hence provides an unmarked means of attenuating pathogenicity but preserving biopesticidal potential in this strain (see Supplementary Discussion, Figure 5). In addition, the BCC0191 $\Delta$ c3 mutant also showed a reduced root colonisation after 14 days compared to the wild type (see Supplementary Discussion, Supplementary Table 7), suggesting it has less potential for bioaccumulation, which is another desirable trait for a biopesticide. Whether $\mathrm{c} 3$ deletion is sufficient to render B. ambifaria as a species completely avirulent remains to be fully determined. B. ambifaria is rarely found in CF lung infections, with a survey of US patients from 1997 to 2007 implicating it collectively with several other B. cepacia complex species as causing $<3 \%$ of all Burkholderia cases31. A 2017 survey of Burkholderia infections in 361 UK CF patients did not find $B$. ambifaria at all32. This epidemiological data combined with the low murine respiratory persistence of $B$. ambifaria (Supplementary Figure $7 \mathrm{~b}$ and Supplementary Figure 7 c) compared to virulent pathogens such as $P$. aeruginosa19,20, suggests that $B$. ambifaria has low pathogenicity. From this start point, attenuation of virulence using unmarked c3 deletion as performed herein, combined with further essential gene mutation strategies as used to construct live bacterial vaccines, could also provide a route towards the development of safe $B$. ambifaria biopesticides.

\section{Conclusion}

Biological control agents have been applied to crops with success in the past, but no in-depth genomic or analytical chemistry analyses have been conducted on individual species to assess their biocontrol potential. This study demonstrated the benefits of using genome mining and in vitro antimicrobial screening to define BGCs that contribute to biocontrol, and enable their use in the rational design of future bacterial biopesticides. The potential of cepacin-producing $B$. ambifaria in protecting economically relevant crop species from attack by oomycete pathogens has been demonstrated. It is clear that $B$. ambifaria has accumulated multiple plant protective BGCs that underpin its historical exploitation as a biopesticide2. With an urgent need to sustain crop protection and agricultural production, yet reduce use of environmentally persistent chemical pesticides, systematically repurposing natural biological control agents such as $B$. ambifaria for biotechnology is a timely alternative solution. 


\section{Methods}

\section{Genome sequencing and replicon assembly}

Genomes used in this study were either sequenced as part of this study or downloaded from public databases. 125-nucleotide and 150-nucleotide paired-end reads were generated for 60 B. ambifaria genomes (Supplementary Table 1) using an Illumina HiSeq 2000 and HiSeq X Ten, respectively. Illumina adaptors were trimmed, read quality assessed and contigs assembled as described in the Supplementary Notes. Genomic contigs were re-arranged and scaffolded into replicons by mapping the contigs against three reference genomes using CONTIGuator v2.7.433. The option to fill gaps using strings of "N" was disabled. Reference genomes were B. ambifaria AMMD (SAMN02598309) and B. ambifaria MC40-6 (SAMN02598385), both obtained from the European Nucleotide Archive; the third reference, B. ambifaria BCC0203, was generated using Pacific Biosciences single molecule real time sequencing. The replicons were manually assessed for any scaffolding errors and corrected when necessary. Completed replicons (c1, c2 and c3) were re-circularised based on genes $d n a A$, parA and parB, respectively, using the software Circlator v1.2.134. The species validity of $B$. ambifaria dataset was defined by calculating the average nucleotide identity (ANI) shared between all available $B$. ambifaria genomes using PyANI v0.2.135. Two sequenced strains from this study (BCC1630 and BCC1638) and one publically available strain (RZ2MS16) were excluded from the dataset, using a 95\% ANI species threshold36. The remaining 64 B. ambifaria strains along with mutant derivatives used in this study are listed in the supplementary data (Supplementary Table 1).

\section{Genome mining and specialized metabolite BGC network analysis}

All bioinformatics analyses were performed using the Cloud Infrastructure for Microbial Bioinformatics (CLIMB) computing resource37. Scaffolded replicons and non-scaffolded contigs were annotated using Prokka v1.12-beta38. Bioinformatics tool antiSMASH v. 3.0.517 detected specialized metabolite BGCs in both scaffolded and non-scaffolded contig sequences. Known pathways that were not detected by antiSMASH were identified with nucleotide-nucleotide BLAST v2.6.0+39. BGCs were dereplicated by clustering nucleotide sequences using pairwise Kmer-matching software Mash v1.1.140; reporting a maximum pvalue and maximum distance of 1 and 0.05 , respectively. The resulting distance matrix was visualized with Cytoscape v3.4.041, applying the Jaccard index, p-value and Mash distance (estimated mutation rate between sequences) as edge attributes. Duplicated edges between nodes and self-loops were removed from the network analysis. The resulting cluster network was further refined by comparing the gene topologies of pathway representatives from network clusters of the same specialized metabolite class; and splitting or merging network clusters where necessary.

\section{Genomic analysis and phylogenomics}

The core and accessory genome of the collective 64 B. ambifaria strains was calculated using Roary v3.7.042 using a 95\% minimum percentage identity for blastp, and core gene threshold of $99 \%$ occurrence across the 64 strains. The core gene alignment generated by Roary was used to construct an approximate-maximum-likelihood core-gene phylogeny with double-precision FastTree v2.1.943. The root position was determined by including the 
outgroup species Burkholderia vietnamiensis G4 (PRJNA10696) (Supplementary Figure 10). Once the root branch point was defined, a second tree was constructed using RAxML v8.2.1144 with General Time Reversible (GTR) substitution and a GAMMA model of rate heterogeneity supported by 100 bootstraps.

\section{Culture conditions and antimicrobial activity screens}

All B. ambifaria strains were grown in tryptic soy broth (TSB) at $37^{\circ} \mathrm{C}$ and aerated overnight, unless stated otherwise. The $64 \mathrm{~B}$. ambifaria strains were screened for production of antimicrobials with antagonistic activity against 14 plant, animal and human pathogens, and other reference species (Supplementary Table 5). A standard overlay assay was used to screen for antimicrobial activity, as previously described3, with the amendment of using 400 $\mu$ l overnight $(\mathrm{O} / \mathrm{N})$ culture of the susceptibility-testing organism per $100 \mathrm{ml}$ half-strength iso-sensitest agar. A strain was considered antagonistic if the zone of inhibition was $>10 \mathrm{~mm}$ in diameter. Antagonism assays with A. alternata and $F$. solani involved the re-suspension of mycelia in $1 \mathrm{ml}$ PBS from 9-day old cultures grown in $50 \mathrm{~mm}$ petri dishes on potato dextrose agar; $320 \mu \mathrm{l}$ of mycelial resuspension was used per $100 \mathrm{ml}$ half-strength isosensitest agar before pouring as an overlay. $10 \times 10 \mathrm{~cm}$ square Petri dishes containing basal salts medium 15 supplemented with $4 \mathrm{~g} / \mathrm{l}$ glycerol (BSM-G) 3 were used to screen six isolates concomitantly, and a replicator used to transfer the bacterial culture onto the agar surface from 96-well plates. B. ambifaria strains were grown on BSM-G for three days at $30^{\circ} \mathrm{C}$. Each 96-well plate stored six strains tested for antimicrobial activity, with $200 \mu \mathrm{O} / \mathrm{N}$ culture grown in TSB broth (Oxoid) per used well, and DMSO at a final concentration of $8 \%$ for $-80^{\circ} \mathrm{C}$ storage. Following chloroform-killing, approximately $25 \mathrm{ml}$ of antimicrobial susceptibility test organism-seeded half-strength iso-sensitest agar was poured over each $10 \times 10 \mathrm{~cm}$ square Petri dish. The plates were incubated at the optimum temperatures of each susceptibility-test organism (Supplementary Table 5). The heatmap of pathogen antagonism was created in statistics software R v3.2.3 via RStudio v0.99.484.

\section{Confirmation of the Burkholderia cepacin A BGC-metabolite link}

Insertional mutagenesis was used to disrupt the expression of the cepacin A pathway. Primers were designed to amplify a 649 bp region of the fatty AMP ligase-encoding gene (Supplementary Table 9), yielding a final product of $707 \mathrm{bp}$. The product was amplified using the Taq PCR Master Mix Kit (Qiagen), and ligated into the suicide vector pGp-omegaTp45 following restriction with $\mathrm{XbaI}$ and $E c o R I$ (NEB). The resulting construct was transformed into competent Escherichia coli SY327 via heat-shock (maintained by trimethoprim selection; $50 \mu \mathrm{g} / \mathrm{ml}$ ), and subsequently introduced into $B$. ambifaria via triparental mating with E. coli HB101 carrying the helper plasmid pRK2013 (kanamycin selection, $50 \mu \mathrm{g} / \mathrm{ml}$ ). The transconjugants were selected using trimethoprim $150 \mu \mathrm{g} / \mathrm{ml}$ and polymyxin $600 \mathrm{U} / \mathrm{ml}$. The presence and correct location of the insertional vector was confirmed by PCR. Comparative-HPLC and antimicrobial activity screens between the wildtype and insertional mutant confirmed the disruption of the cepacin BGC.

\section{Construction and phenotypic analysis of a B. ambifaria BCC0191 $\triangle \mathrm{c} 3$ mutant}

The B. ambifaria BCC0191 $\Delta \mathrm{c} 3$ mutant was constructed following the methods outlined in Agnoli et al. 201212. This involved using the pMiniC3 vector, a $12.6 \mathrm{~kb}$ plasmid constructed 
from the origin of replication of the B. cenocepacia $\mathrm{H} 111$ third replicon and containing its $\operatorname{rep} A, \operatorname{par} B$, and $\operatorname{par} A$ genes, and trimethoprim resistance and sucrose counter selection cassettes12. In brief, the pMiniC3 vector was mated into the BCC0191 wild-type to displace the native $\mathrm{c} 3$ replicon via a tri-parental mating involving the donor $E$. coli $\mathrm{MC} 1061$ pMiniC3, recipient B. ambifaria BCC0191 and E. coli HB101 carrying the helper plasmid pRK2013. B. ambifaria BCC0191 pMinic3 clones were subsequently cured of pMinic3 by sucrose counter-selection. B. ambifaria BCC0191 $\Delta \mathrm{c} 3$ clones were screened for the absence of both replicon c3 and plasmid pMinic3 by PCR using the DreamTaq Green PCR Master Mix (2X) (Thermo Scientific) (Supplementary Table 9). The virulence of $B$. ambifaria BCC0191 and its $\Delta \mathrm{c} 3$ mutant was evaluated in $G$. mellonella wax moth larvae12 and murine chronic lung 19,20 infection models as described below.

\section{Detection of LuxR homologues as specialized metabolite regulators}

LuxR-encoding gene homologues were identified by replicating the systematic in silico approach previously described46. In brief, a Hidden Markov Model was built to identify potential LuxR-encoding genes; and these candidates were annotated for encoded protein domains. Candidate genes were considered LuxR-encoding if all four conserved LuxR protein domains were detected. The extracted amino acid sequences were aligned using MAFFT v7.305b47 and a phylogenetic tree generated using FastTree v2.1.943. The regulatory function of the luxR gene was inferred either from the literature or genes with putative functions starting within $5 \mathrm{kbp}$ upstream and downstream of the luxR gene.

\section{Culture conditions, extraction protocol and high resolution mass spectrometry}

All B. ambifaria strains were grown at $30^{\circ} \mathrm{C}$ on BSM-G. The original cepacin producer, strain ATCC 395396, was obtained from the Belgium Coordinated Collection of Microorganisms where it is deposited as B. diffusa strain LMG 24093. Single plates were extracted by addition of $4 \mathrm{ml}$ of acetonitrile for 2 hours, followed by centrifugation to remove debris. Crude extracts were directly analysed by UHPLC-ESI-Q-TOF-MS. UHPLCESI-Q-TOF-MS analyses were performed using a Dionex UltiMate 3000 UHPLC connected to a Zorbax Eclipse Plus C-18 column $(100 \times 2.1 \mathrm{~mm}, 1.8 \mu \mathrm{m})$ coupled to a Bruker MaXis II mass spectrometer. Mobile phases consisted of water (A) and acetonitrile (B), each supplemented with $0.1 \%$ formic acid. A gradient of 5\% B to $100 \%$ B over 30 minutes was employed at a flow rate of $0.2 \mathrm{ml} / \mathrm{min}$. The mass spectrometer was operated in positive ion mode with a scan range of 50-3000 m/z. Source conditions were: end plate offset at $-500 \mathrm{~V}$; capillary at $-4500 \mathrm{~V}$; nebulizer gas $\left(\mathrm{N}_{2}\right)$ at 1.6 bar; dry gas $\left(\mathrm{N}_{2}\right)$ at $8 \mathrm{~L} \mathrm{~min}^{-1}$; dry temperature at $180^{\circ} \mathrm{C}$. Ion transfer conditions were: ion funnel RF at $200 \mathrm{Vpp}$; multiple RF at $200 \mathrm{Vpp}$; quadrupole low mass at $55 \mathrm{~m} / \mathrm{z}$; collision energy at $5.0 \mathrm{eV}$; collision $\mathrm{RF}$ at 600 $\mathrm{Vpp}$; ion cooler RF at 50-350 Vpp; transfer time at $121 \mu$ s; pre-pulse storage time at $1 \mu \mathrm{s}$. Calibration was performed with $1 \mathrm{mM}$ sodium formate through a loop injection of $20 \mu \mathrm{L}$ at the start of each run.

\section{Biocontrol of Pythium ultimum using a Pisum sativum model}

The infestation of soil with Pythium ultimum Trow var. ultimum (MUCL 16164) was developed from the methodology proposed in Toda et al. 201548. Plugs of $P$. ultimum were grown at approximately $22^{\circ} \mathrm{C}$ on potato dextrose agar (PDA) for three days. Infested soil 
was produced by mixing the surface mycelia from the PDA agar plates into a 5:1 compost:sand mixture (one-90 mm PDA petri per $120 \mathrm{~g}$ soil), and incubating the soil at approximately $22^{\circ} \mathrm{C}$ for three days. Potting mix was composed of $1 \%(\mathrm{w} / \mathrm{w})$ Pythiuminfested soil in unsterilised non-infested soil, or $100 \%$ unsterilised non-infested soil for a non-infested control. Unsterilised $P$. sativum seeds (Early Onward cultivar) were coated with $B$. ambifaria before planting. $B$. ambifaria coating suspension was produced as follows: an overnight (approximately 18 hours) TSB culture of $B$. ambifaria was washed in sterile $1 \mathrm{x}$ volume phosphate buffer solution (PBS) and re-suspended in sterile $0.5 \mathrm{x}$ volume PBS. The suspension was adjusted to $10 \mathrm{x} 0.5 \mathrm{OD}$ at $600 \mathrm{~nm}$ (approximately $10^{9} \mathrm{cfu} / \mathrm{ml}$ ), and either applied at the neat concentration or diluted to achieve the desired inoculum levels $\left(10^{8}\right.$ and $\left.10^{7} \mathrm{cfu} / \mathrm{ml}\right)$. Control $P$. sativum seeds lacking the $B$. ambifaria coating were dipped in PBS. $P$. sativum plants were grown at $22^{\circ} \mathrm{C}$ with a $16-\mathrm{h}$ light $/ 8$-h dark photoperiod $(70 \% \mathrm{RH})$ for 14 days, and watered as required. Groups of ten seeds per inoculum and seed coat organism were assayed per experiment, and the experiment was performed in triplicate. Significant differences between BCC0191 wild-type and mutant derivatives was assessed using two tailed t-test or Welch's two tailed t-test. Two tailed t-test assumptions were normally distributed data (Shapiro-Wilk test) and equal variances (Bartlett test); Welch's two tailed ttest did not assume equal variances.

\section{Metabolite extraction and HPLC analysis}

To confirm the absence of cepacin A in the BGC mutants constructed for the different $B$. ambifaria strain backgrounds (BCC0191, BCC1252, BCC1241, BCC0477, BCC1259 and BCC1218; Supplementary Figure 3), each strain was grown on BSM-G agar for three days at $22^{\circ} \mathrm{C}$, the bacterial growth removed and an equal amount of agar (a $2 \mathrm{~cm}$ disc) extracted with $0.5 \mathrm{ml}$ ethyl acetate for 2 hours at room temperature. This was fractionated on an acetonitrile gradient (5\% to 95\%) using a Waters ${ }^{\circledR}$ AutoPurification ${ }^{\mathrm{TM}}$ HPLC System fitted with a reverse phase analytical column (Waters® XSelect CSH C18, 4.6 x $100 \mathrm{~mm}, 5 \mu \mathrm{m}$ ). Metabolites eluted from the column were detected by a photodiode array and the peak corresponding to cepacin A identified by its retention time in relation to the LC-MS confirmed presence of the polyyne in this fraction.

\section{In vivo killing assay using Galleria wax moth larvae}

Galleria mellonella wax moth larvae were sourced from BioSystems Technology Ltd TruLarv (Exeter, UK). Bacterial cultures of BCC0191 wild-type and BCC0191 $\Delta \mathrm{c} 3$ were grown overnight (approximately 18 hours) in TSB broth at $37^{\circ} \mathrm{C}$, washed and re-suspended in $1 \mathrm{x}$ volume PBS before being adjusted to approximately $1 \times 10^{6} \mathrm{cfu} / \mathrm{ml}$. Larvae were injected in the hindmost proleg on the right-side of the abdomen. Each larva was injected with $10 \mu \mathrm{l}$ aliquots of $B$. ambifaria BCC0191 wild-type and BCC0191 $\Delta \mathrm{c} 3$ bacterial suspensions with a 25G x 1" needle (BD Microlance 3) using a 1705TLL, $50 \mu \mathrm{L}$ syringe (Hamilton). PBS injections were included as controls. Each condition included ten larvae, and the experiment was performed in triplicate over three days. The larvae were incubated at $37^{\circ} \mathrm{C}$ for 72 hours, and their survival status was monitored at 18, 24, 42, 48, 66 and 72 hours post-inoculation. Larvae were recorded as dead when they failed to respond to physical agitation. The total viable count of the bacterial suspensions was calculated during the first 
and third replicate via drop-count. The mean average $\mathrm{cfu} / \mathrm{ml}$ of BCC0191 wild-type and BCC0191 $\Delta \mathrm{c} 3$ suspensions were $1.5 \times 10^{6} \mathrm{cfu} / \mathrm{ml}$ and $1.0 \times 10^{6} \mathrm{cfu} / \mathrm{ml}$, respectively.

Murine chronic lung infection model-A murine respiratory infection model as used for modelling $P$. aeruginosa pathogenicity was applied to $B$. ambifaria essentially as described19,20. All experiments were carried out at the University of Liverpool with approval from the UK Home Office and University ethics committee. Randomisation of mice to cages (experimental groups) was performed by animal unit staff with no role in study design as described19. An initial dosing experiment demonstrated good tolerance of an infectious dose of $10^{6} \mathrm{~B}$. ambifaria, equivalent to that used for $P$. aeruginosa in previous studies 19,20. Sample size was calculated to give $95 \%$ power at alpha 0.05 to detect a $>4$ fold difference in lung CFU between BCC0191 and BCC0191 $\triangle \mathrm{c} 3$, assuming mean CFU of 100 in BCC0191 lung and a standard deviation of $50 \%$ of the mean (as determined in preliminary experiments). Groups of 6 mice (female BALB/c, 6-8 weeks old; Charles River, $\mathrm{UK})$ were infected intranasally under anaesthesia $\left(\mathrm{O}_{2} /\right.$ isolfuorane), achieving an actual dose of $2 \times 10^{6}$ B. ambifaria BCC0191 or its third chromosome replicon mutant BCC0191 $\Delta \mathrm{c} 3$. Symptoms of disease were monitored, and mice culled at 24 hours, 48 hours and 5 days post infection. Researchers were not blinded to the experimental groupings. The nasopharyngeal tissue, lungs and spleens were removed, homogenised in $3 \mathrm{ml}$ PBS, serial dilutions prepared and plated onto Burkholderia cepacia selective agar (Oxoid, UK) for enumeration of surviving $B$. ambifaria. No animals were excluded from the analysis. Colonies from the tissue of mice carrying infection were confirmed to be strain B. ambifaria BCC0191 by Random Amplified Polymorphic DNA typing (RAPD; see Supplementary Figure 8).

\section{Rhizocompetence of B. ambifaria BCC0191 WT and derivatives}

The rhizocompetence of B. ambifaria BCC0191 and its derived mutants were evaluated essentially as described49. Liquid cultures of BCC0191, BCC0191::ccnJ and BCC01911 $\Delta$ c3 were grown in TSB broth overnight at $37^{\circ} \mathrm{C}(50 \mu \mathrm{g} / \mathrm{ml}$ trimethoprim for BCC0191::ccnJ), then washed and re-suspended in 1x volume PBS and adjusted to $1 \times 10^{9} \mathrm{cfu} / \mathrm{ml}$. Pisum sativum (pea) seeds were coated in the bacterial suspensions and planted in a potting mix composed of 5:1 multi-purpose compost to sand. The seeds were germinated and grown at $22^{\circ} \mathrm{C}$ with a 16 hour light -8 hour dark photoperiod (70\% RH) for 14 days and watered as required. After 14 days the plants were removed, and excess soil shaken from the root systems. The $1^{\text {st }}-2^{\text {nd }} \mathrm{cm}$ of root was excised, macerated in $1 \mathrm{ml}$ PBS, and serially diluted. Dilutions of the root suspension were plated onto Burkholderia cepacia selective agar (BCSA; Oxoid UK) and incubated at $37^{\circ} \mathrm{C}$ for 24 hours to calculate total viable counts. Three plants were used per strain derivative, and three un-inoculated seeds included as a control. Any growth from the control plants was subject to RAPD PCR profiling to distinguish the bacteria from $B$. ambifaria. Non- $B$. ambifaria growth on the control plates was discounted from the total viable counts. Total viable counts were standardised to $1 \mathrm{~g}$ fresh weight of root.

\section{Supplementary Material}

Refer to Web version on PubMed Central for supplementary material. 


\section{Acknowledgements}

AM is funded by a Biotechnology and Biological Sciences Research Council (BBSRC) South West doctoral training partnership award (BY1910 7007). EM, GC, TC and JP acknowledge additional support for genome mining from BBSRC award BB/L021692/1; CJ and MJ were funded by this award. M.J is currently the recipient of a BBSRC Future Leader Fellowship (BB/R01212/1). The Bruker maXis II UHPLC-ESI-Q-TOF-MS system used in this research was funded by the BBSRC (BB/M017982/1). GW was supported by awards to EM from the Life Sciences Bridging Fund and Wellcome Trust Institutional Strategic Support Fund held at Cardiff University. TRC and MB acknowledge funding support from the Medical Research Council's Cloud Infrastructure for Microbial Bioinformatics (MR/L015080/1) which provided the computational resources to undertake the analyses for this work. DN and AG acknowledge funding from a Wellcome Trust and Royal Society Sir Henry Dale Fellowship awarded to DN (Grant Number 204457/Z/16/Z). GC is the recipient of a Wolfson Research Merit Award from the Royal Society (WM130033). We thank Leo Eberl and Kirsty Agnoli for provision of mini-c3 used for third replicon deletion.

\section{References}

1. Depoorter E, et al. Burkholderia: an update on taxonomy and biotechnological potential as antibiotic producers. Appl Microbiol Biotechnol. 2016; 100:5215-5229. [PubMed: 27115756]

2. Parke JL, Gurian-Sherman D. Diversity of the Burkholderia cepacia complex and implications for risk assessment of biological control strains. Annu Rev Phytopathol. 2001; 39:225-258. [PubMed: 11701865]

3. Mahenthiralingam E, et al. Enacyloxins are products of an unusual hybrid modular polyketide synthase encoded by a cryptic Burkholderia ambifaria genomic island. Chem Biol. 2011; 18:665677. [PubMed: 21609847]

4. Masschelein J, Jenner M, Challis GL. Antibiotics from Gram-negative bacteria: a comprehensive overview and selected biosynthetic highlights. Nat Prod Rep. 2017; 34:712-783. [PubMed: 28650032]

5. Seyedsayamdost MR, et al. Quorum-sensing-regulated bactobolin production by Burkholderia thailandensis E264. Org Lett. 2010; 12:716-719. [PubMed: 20095633]

6. Song L, et al. Discovery and biosynthesis of gladiolin: A Burkholderia gladioli antibiotic with promising activity against Mycobacterium tuberculosis. J Am Chem Soc. 2017; 139

7. Flórez LV, et al. Antibiotic-producing symbionts dynamically transition between plant pathogenicity and insect-defensive mutualism. Nat Commun. 2017; 8:15172. [PubMed: 28452358]

8. Kim J, et al. Quorum sensing and the LysR-type transcriptional activator ToxR regulate toxoflavin biosynthesis and transport in Burkholderia glumae. Mol Microbiol. 2004; 54:921-934. [PubMed: 15522077]

9. Ross C, Scherlach K, Kloss F, Hertweck C. The molecular basis of conjugated polyyne biosynthesis in phytopathogenic bacteria. Angew Chemie - Int Ed. 2014; 53:7794-7798.

10. Mansfield J, et al. Top 10 plant pathogenic bacteria in molecular plant pathology. Mol Plant Pathol. 2012; 13:614-629. [PubMed: 22672649]

11. Howden AJM, Rico A, Mentlak T, Miguet L, Preston GM. Pseudomonas syringae pv. syringae B728a hydrolyses indole-3-acetonitrile to the plant hormone indole-3-acetic acid. Mol Plant Pathol. 2009; 10:857-865. [PubMed: 19849791]

12. Agnoli K, et al. Exposing the third chromosome of Burkholderia cepacia complex strains as a virulence plasmid. Mol Microbiol. 2012; 83:362-378. [PubMed: 22171913]

13. Schmidt $S$, et al. Production of the antifungal compound pyrrolnitrin is quorum sensing-regulated in members of the Burkholderia cepacia complex. Environ Microbiol. 2009; 11:1422-1437. [PubMed: 19220396]

14. Tawfik KA, et al. Burkholdines 1097 and 1229, potent antifungal peptides from Burkholderia ambifaria 2.2N. Org Lett. 2010; 12:664-666. [PubMed: 20085289]

15. Hareland WA, Crawford RL, Chapman PJ, Dagley S. Metabolic function and properties of 4hydroxyphenylacetic acid 1-hydroxylase from Pseudomonas acidovorans. J Bacteriol. 1975; 121:272-85. [PubMed: 234937]

16. Parker WL, et al. Cepacin A and cepacin B, two new antibiotics produced by Pseudomonas cepacia. J Antibiot (Tokyo). 1984; 37:431-440. [PubMed: 6547430] 
17. Weber T, et al. antiSMASH 3.0-a comprehensive resource for the genome mining of biosynthetic gene clusters. Nucleic Acids Res. 2015; 43:W237-43. [PubMed: 25948579]

18. Ishida K, Lincke T, Behnken S, Hertweck C. Induced Biosynthesis of Cryptic Polyketide Metabolites in a Burkholderia thailandensis Quorum Sensing Mutant. J Am Chem Soc. 2010; 132:13966-13968. [PubMed: 20853892]

19. Fothergill JL, Neill DR, Loman N, Winstanley C, Kadioglu A. Pseudomonas aeruginosa adaptation in the nasopharyngeal reservoir leads to migration and persistence in the lungs. Nat Commun. 2014; 5:4780. [PubMed: 25179232]

20. Bricio-Moreno L, et al. Evolutionary trade-offs associated with loss of PmrB function in hostadapted Pseudomonas aeruginosa. Nat Commun. 2018; 9:2635. [PubMed: 29980663]

21. Duerkop BA, et al. Quorum-sensing control of antibiotic synthesis in Burkholderia thailandensis. J Bacteriol. 2009; 191:3909-3918. [PubMed: 19376863]

22. Lee J, Zhang L. The hierarchy quorum sensing network in Pseudomonas aeruginosa. Protein Cell. 2015; 6:26-41. [PubMed: 25249263]

23. Yabuuchi E, et al. Proposal of Burkholderia gen. nov. and transfer of seven species of the genus Pseudomonas homology group II to the new genus, with the type species Burkholderia cepacia (Palleroni and Holmes 1981) comb. nov. Microbiol Immunol. 1992; 36:1251-75. [PubMed: 1283774]

24. Vanlaere E, et al. Burkholderia latens sp. nov., Burkholderia diffusa sp. nov., Burkholderia arboris sp. nov., Burkholderia seminalis sp. nov. and Burkholderia metallica sp. nov., novel species within the Burkholderia cepacia comple. Int J Syst Evol Microbiol. 2008; 58:1580-1590. [PubMed: 18599699]

25. Fritsche K, et al. Biosynthetic genes and activity spectrum of antifungal polyynes from Collimonas fungivorans Ter331. Environ Microbiol. 2014; 16:1334-1345. [PubMed: 24588891]

26. Kai K, Sogame M, Sakurai F, Nasu N, Fujita M. Collimonins A-D, Unstable Polyynes with Antifungal or Pigmentation Activities from the Fungus-Feeding Bacterium Collimonas fungivorans Ter331. Org Lett. 2018; 20:3536-3540. [PubMed: 29792438]

27. Haas D, Keel C. Regulation of antibiotic production in root-colonizing Pseudomonas spp. And relevance for biological control of plant disease. Annu Rev Phytopathol. 2003; 41:117-153. [PubMed: 12730389]

28. Palazzini JM, Dunlap CA, Bowman MJ, Chulze SN. Bacillus velezensis RC 218 as a biocontrol agent to reduce Fusarium head blight and deoxynivalenol accumulation: Genome sequencing and secondary metabolite cluster profiles. Microbiol Res. 2016; 192:30-36. [PubMed: 27664721]

29. Law JW-F, et al. The potential of Streptomyces as biocontrol agents against the Rice Blast Fungus, Magnaporthe oryzae (Pyricularia oryzae). Front Microbiol. 2017; 8:3. [PubMed: 28144236]

30. Eberl L, Vandamme P. Members of the genus Burkholderia: good and bad guys. F1000Research. 2016; 5

31. LiPuma JJ. The Changing Microbial Epidemiology in Cystic Fibrosis. Clin Microbiol Rev. 2010; 23:299-323. [PubMed: 20375354]

32. Kenna DTD, et al. Prevalence of Burkholderia species, including members of Burkholderia cepacia complex, among UK cystic and non-cystic fibrosis patients. J Med Microbiol. 2017; 66:490-501. [PubMed: 28463663]

33. Galardini M, Biondi EG, Bazzicalupo M, Mengoni A. CONTIGuator: a bacterial genomes finishing tool for structural insights on draft genomes. Source Code Biol Med. 2011; 6:11. [PubMed: 21693004]

34. Li H. Circlator: automated circularization of genome assemblies using long sequencing reads. $2013 ; 16$

35. Pritchard L, et al. Genomics and taxonomy in diagnostics for food security: soft-rotting enterobacterial plant pathogens. Anal Methods. 2016; 8:12-24.

36. Richter M, Rossello-Mora R. Shifting the genomic gold standard for the prokaryotic species definition. Proc Natl Acad Sci. 2009; 106:19126-19131. [PubMed: 19855009]

37. Connor TR, et al. CLIMB (the Cloud Infrastructure for Microbial Bioinformatics): an online resource for the medical microbiology community. Microb Genomics. 2016; 2 
38. Seemann T. Prokka: rapid prokaryotic genome annotation. Bioinformatics. 2014; 30:2068-2069. [PubMed: 24642063]

39. Morgulis A, et al. BLAST+: architecture and applications. Bioinformatics. 2008; 24:1757-1764. [PubMed: 18567917]

40. Ondov BD, et al. Mash: fast genome and metagenome distance estimation using MinHash. Genome Biol. 2016; 17:132. [PubMed: 27323842]

41. Shannon P, et al. Cytoscape: A software environment for integrated models of biomolecular interaction networks. Genome Res. 2003; 13:2498-2504. [PubMed: 14597658]

42. Page AJ, et al. Roary: rapid large-scale prokaryote pan genome analysis. Bioinformatics. 2015; 31:3691-3693. [PubMed: 26198102]

43. Price MN, Dehal PS, Arkin AP. FastTree 2 - Approximately maximum-likelihood trees for large alignments. PLoS One. 2010; 5:e9490. [PubMed: 20224823]

44. Stamatakis A. RAxML version 8: a tool for phylogenetic analysis and post-analysis of large phylogenies. Bioinformatics. 2014; 30:1312-3. [PubMed: 24451623]

45. Flannagan RS, Aubert D, Kooi C, Sokol PA, Valvano MA. Burkholderia cenocepacia requires a periplasmic HtrA protease for growth under thermal and osmotic stress and for survival in vivo. Infect Immun. 2007; 75:1679-1689. [PubMed: 17220310]

46. Gan HM, et al. Whole genome sequencing and analysis reveal insights into the genetic structure, diversity and evolutionary relatedness of $l u x I$ and $l u x R$ homologs in bacteria belonging to the Sphingomonadaceae family. Front Cell Infect Microbiol. 2014; 4:188. [PubMed: 25621282]

47. Katoh K, Misawa K, Kuma K, Miyata T. MAFFT: a novel method for rapid multiple sequence alignment based on fast Fourier transform. Nucleic Acids Res. 2002; 30:3059-66. [PubMed: 12136088]

48. Toda T, Iwasa A, Fuji S, Furuya H. Widespread occurrence of Pythium arrhenomanes pathogenic to rice seedlings around Japanese rice fields. Plant Dis. 2015; 99:1823-1831. [PubMed: 30699504]

49. Vidal-Quist JC, et al. Arabidopsis thaliana and Pisum sativum models demonstrate that root colonization is an intrinsic trait of Burkholderia cepacia complex bacteria. Microbiology. 2014; 160:373-384. [PubMed: 24327425]

50. Hadfield J, et al. Phandango: an interactive viewer for bacterial population genomics. Bioinformatics. 2018; 34:292-293. 


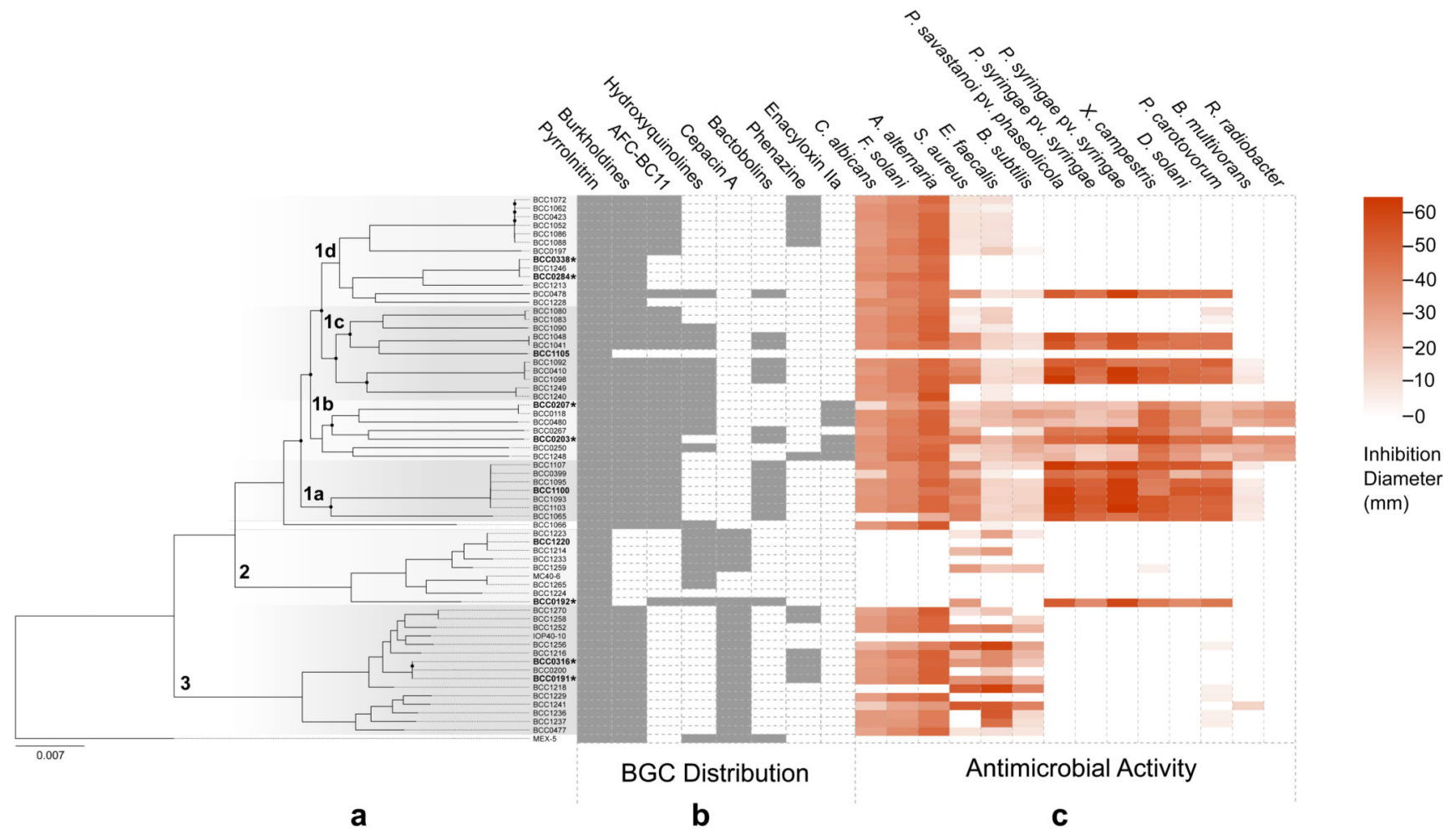

Figure 1. Core-gene phylogeny of $64 \mathrm{~B}$. ambifaria strains (a) aligned with presence/absence grid of known antimicrobial specialized metabolite BGCs (b) and antimicrobial activity heatmap (c). (a) The phylogenetic tree was constructed based on 3784 core genes identified and aligned using the software Roary. The root was determined using a secondary tree containing an outgroup species, Burkholderia vietnamiensis G4 (Supplementary Figure 10). Six clades were defined in the phylogeny, however, strains BCC1066 and MEX-5 branched outside these clades. Strains subject to further LC-MS analysis are highlighted in bold; strains with historical biocontrol usage are indicated with an asterisk. RAxML was used to construct the maximum-likelihood phylogeny using the generalised time reversible (GTR) model with a GAMMA substitution (100 bootstraps). Nodes with bootstrap values $<70 \%$ are indicated with black circles. The evolutionary distance scale bar represents the number of base substitutions per site. (b) The presence of the eight characterised anti-fungal and antibiotic gene clusters: pyrrolnitrin, burkholdine, AFC-BC11, hydroxyquinolines, cepacin A, bactobolins, phenazine and enacyloxin IIa in the $64 \mathrm{~B}$. ambifaria strains are ordered by phylogenetic position. Matrix generated using Phandango50. (c) The antimicrobial activity of $62 \mathrm{~B}$. ambifaria strains were defined by measuring the diameter of the zones of inhibition $(\mathrm{mm}) ; n=2$ overlays of each $B$. ambifaria strain against each susceptibility organism. Heatmap shows mean zone of inhibition. Strains MEX-5 and IOP40-10 were not available for the antimicrobial production assay. 
Core Specialised Metabolite BGCs

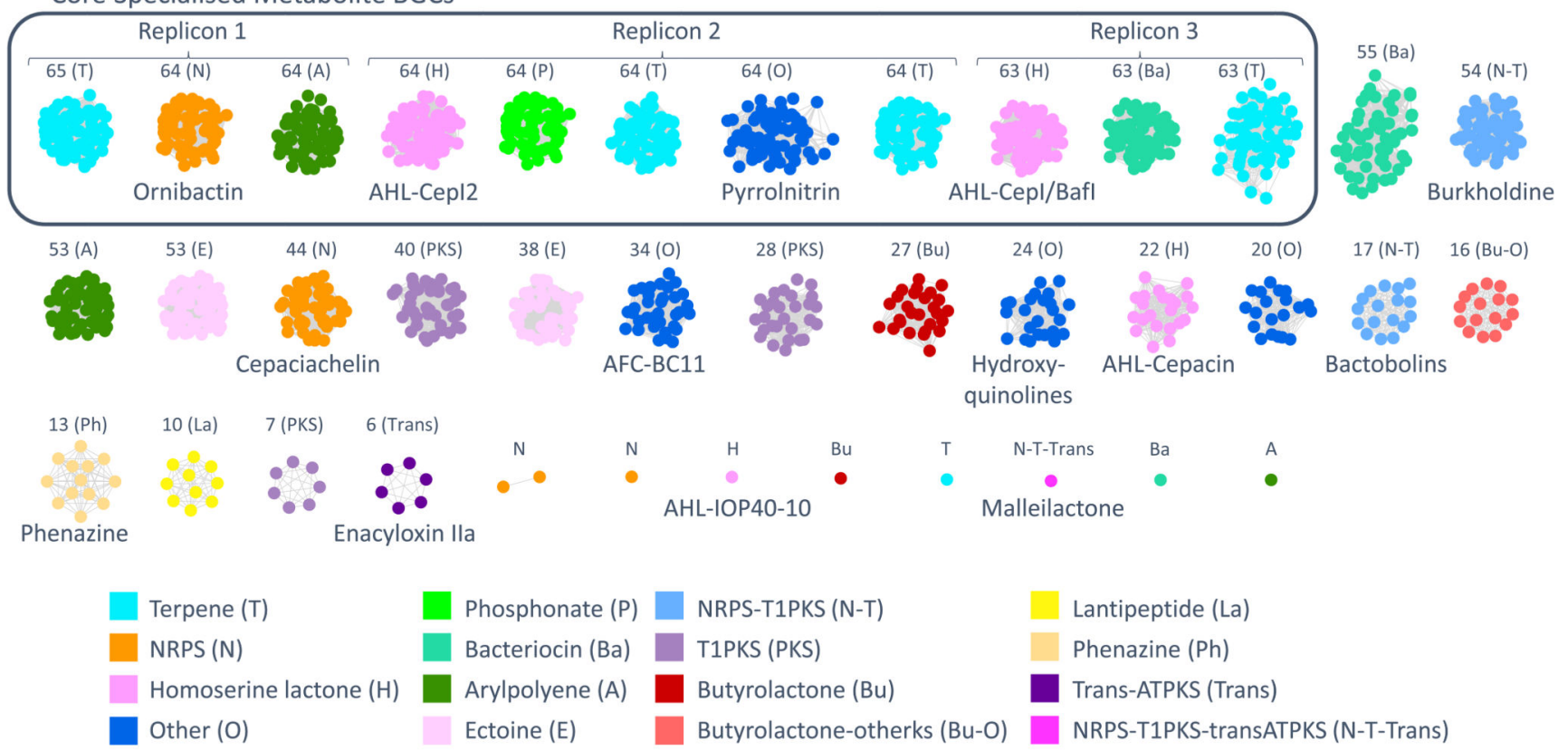

Figure 2. Specialized metabolite BGC network analysis of $64 \mathrm{~B}$. ambifaria strains.

A total of 1,272 BGCs were detected across the 64 strains, and dereplication indicated these represented 38 distinct BGCs (38 distinct network clusters). Nucleotide sequences were clustered using Mash and visualized with Cytoscape. This network analysis was used to provide a visual summary of the breadth of $B$. ambifaria BGCs including their biosynthetic diversity, strain distribution, and core or accessory nature within the species. Each node represents a specialized metabolite BGC extracted from a single $B$. ambifaria strain. Node colours represent specialized metabolite classes, and numbers correspond to the number BGC examples (nodes) of each distinct BGC (network cluster). Core BGCs were defined as BGCs that occurred in $>98 \%$ of $B$. ambifaria strains. Characterised BGCs known in the literature are labelled. BGCs responsible for pyrrolnitrin, AFC-BC11 and hydroxyquinolines biosynthesis are classified as Other $(\mathrm{O})$ by antiSMASH but represent different metabolite classes not recognised by antiSMASH. 


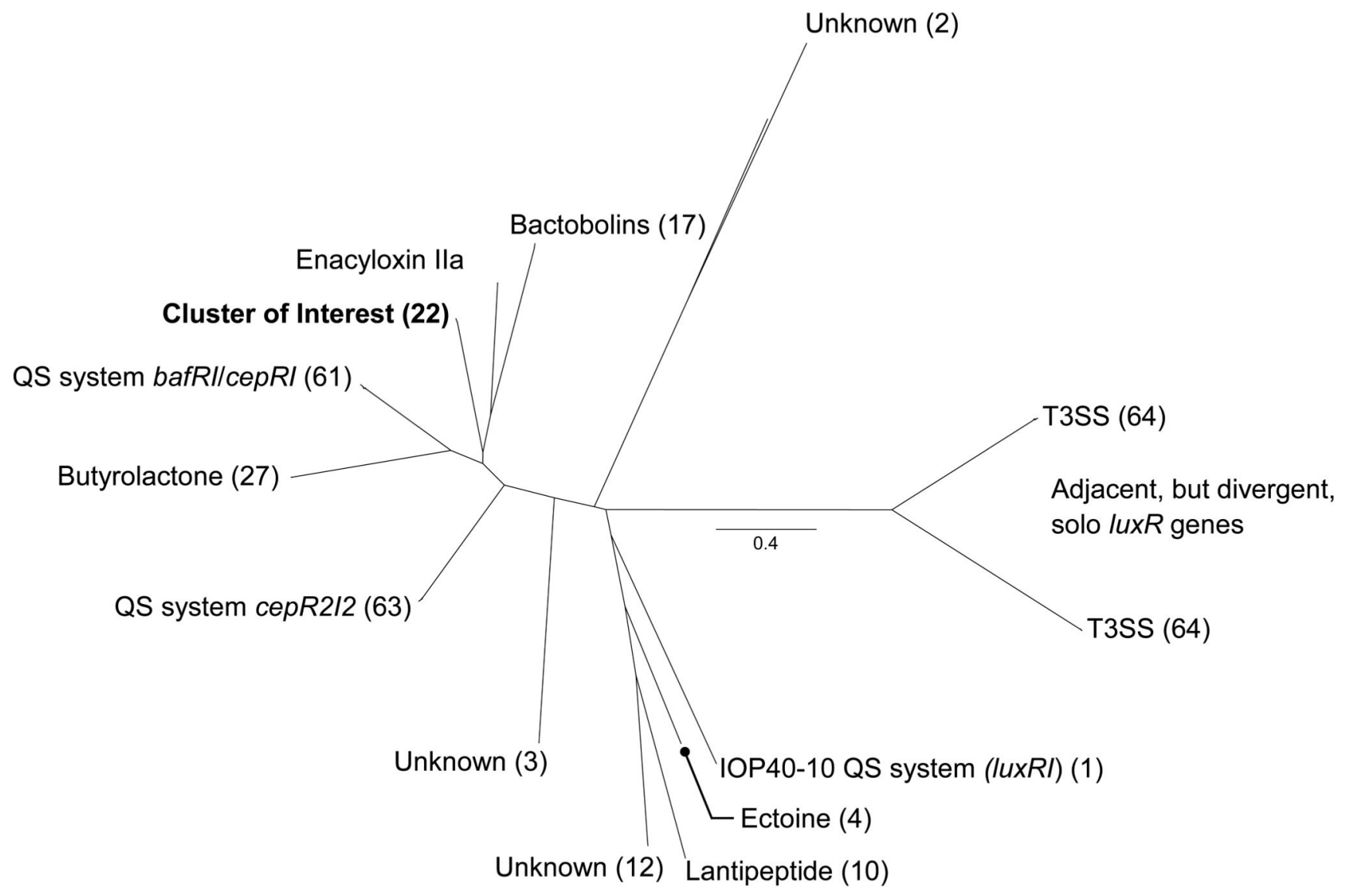

Figure 3. Unrooted phylogeny of LuxR protein homologues extracted from 64 B. ambifaria strains.

Branches were labelled with characterised quorum sensing systems or putative/confirmed LuxR regulatory functions based on the literature and annotated flanking genes starting within $5 \mathrm{kbp}$ upstream and/or downstream of the $1 u x R$ gene. The number of strains encoding distinct LuxR homologues is indicated in brackets. A total of 356 homologues were identified across the 64 strains, representing 14 distinct LuxR protein clades. FastTree was used to construct the approximate-maximum-likelihood phylogeny using the generalised time reversible substitution model. The evolutionary distance scale bar represents the number of base substitutions per site. 
a
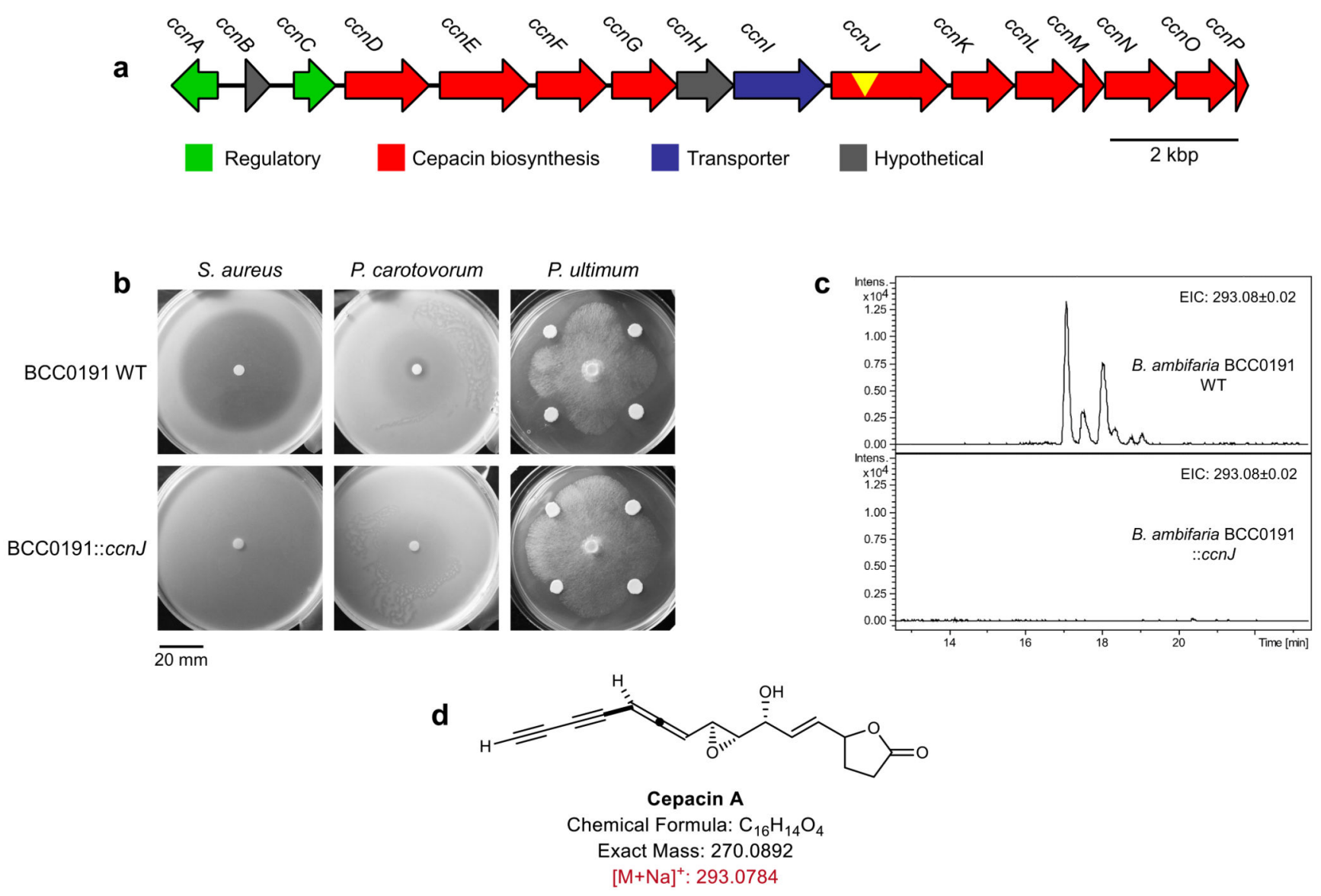

Figure 4. Organization of the cepacin A biosynthetic gene cluster, LC-MS analysis of cepacin A production and antimicrobial screening of B. ambifaria BCC0191 wild-type (WT) and cepacin A deficient derivative $(:: c c n J)$.

(a) Organisation and putative function of genes within the cepacin A BGC; further annotation details are provided in Supplementary Figure 9. The insertion site of the vector used during mutagenesis is highlighted by the inverted yellow triangle. (b) Zones of inhibition against $S$. aureus NCTC 12981, P. carotovorum LMG 2464 and P. ultimum Trow var. ultimum MUCL 16164 by BCC0191 WT and BCC0191::ccnJ. Scale bar represents 20 mm. $n=3$ biological replicates. Images were converted to greyscale, brightness decreased by $20 \%$, and contrast increased by $20 \%$. (c) Extracted ion chromatograms at $\mathrm{m} / \mathrm{z}=293.08$ \pm 0.02 , corresponding to $[\mathrm{M}+\mathrm{Na}]^{+}$for cepacin A, from LC-MS analyses of crude extracts from agar-grown cultures of BCC0191 WT (top) and the BCC0191::ccnJ mutant (bottom); $n$ $=3$ independent LC-MS analyses of WT and mutant cultures. (d) Structure of cepacin A, the identity of which was confirmed by comparison to an authentic standard from a known producer (Supplementary Figure 3). 
a) B. ambifaria WT vs ::ccnJ

\section{BCC0191 WT}

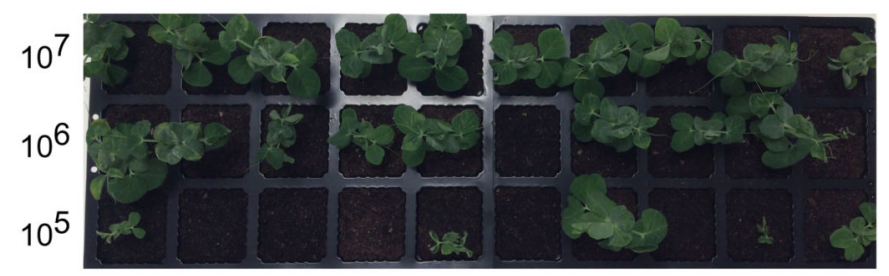

BCC0191::ccnJ

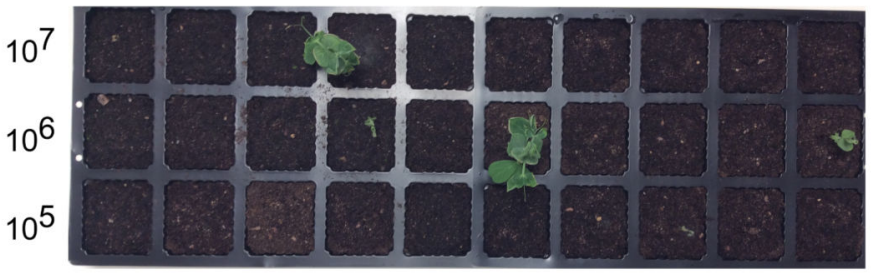

b) B. ambifaria WT vs $\Delta \mathrm{c} 3$

\section{BCC0191 WT}

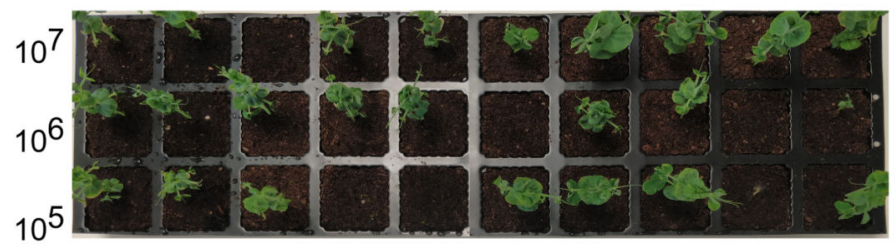

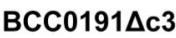

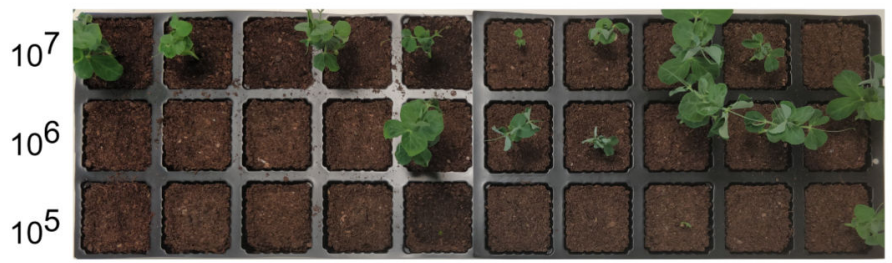

\% Survival of $P$. sativum plants

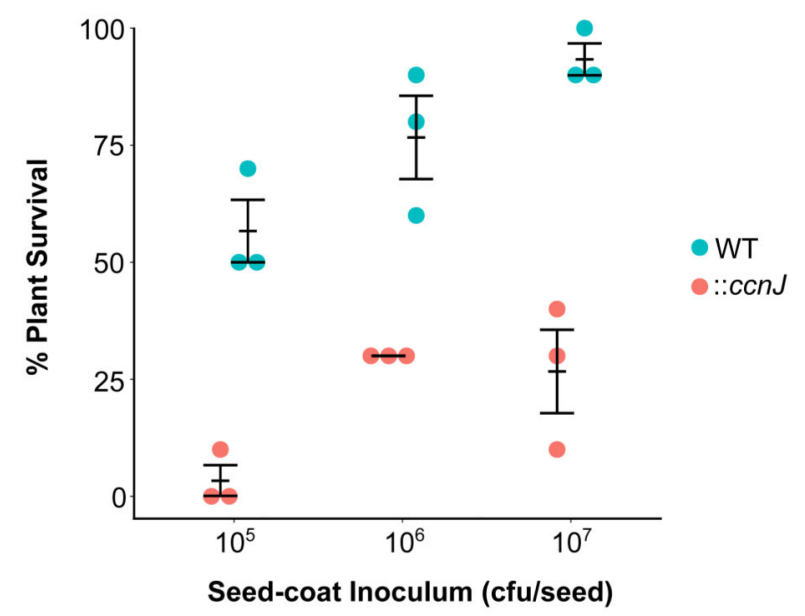

\% Survival of $P$. sativum plants

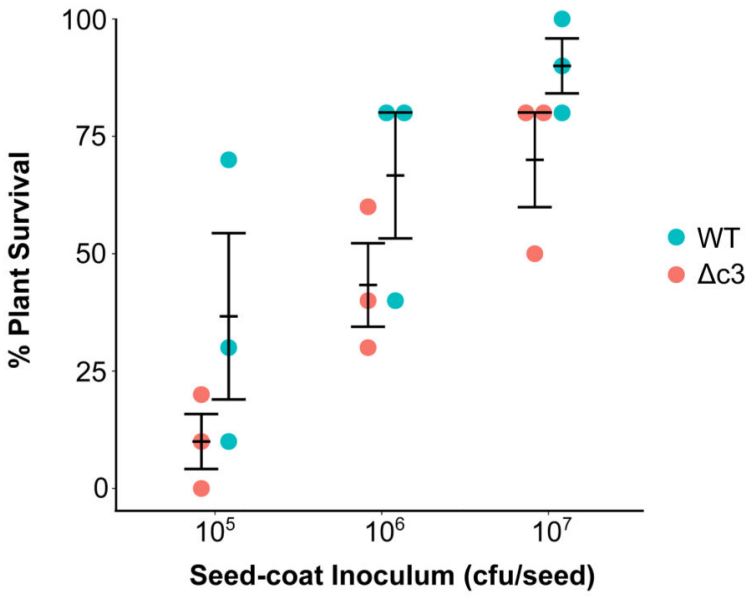

Figure 5. Biological control of Pythium damping-off disease is mediated by B. ambifaria cepacin. (a) Pea germination (14 days) in P. ultimum infested soil observed for groups of 10 seeds coated with $10^{7}, 10^{6}$ and $10^{5} \mathrm{cfu}$, respectively, of BCC0191 wild-type (WT) and

BCC0191::ccnJ. The overall percentage survival of germinating peas treated with the WT and BCC0191::ccnJ B. ambifaria strains is shown on the right of panel A. Survival was assessed as plants that had stems $>30 \mathrm{~mm}$ in height after 14 days. Plant survival was significantly different at every inoculum level between BCC0191 WT and BCC0191::ccnJ, as indicated by two-sided t-test or Welch's two-sided t-test $(*=\mathrm{p}<0.05 ; * *=\mathrm{p}<0.01)$; significant difference (left to right) $\mathrm{p}=0.002, \mathrm{p}=0.03, \mathrm{p}=0.002$ with $95 \%$ confidence interval. $n=10$ seeds per condition (seed coat) and dosage (cfu/seed), repeated in triplicate. Centre bar represents mean, and error bars represent standard error. (b) Pea germination (14 days) in P. ultimum infested soil observed for groups of 10 seeds coated with $10^{7}, 10^{6}$ and 
$10^{5} \mathrm{cfu}$, respectively, of BCC0191 WT and BCC0191 $\Delta \mathrm{c} 3$. The overall percentage survival of germinating peas treated with BCC0191 WT and BCC0191 $\Delta \mathrm{c} 3$ is shown on the right of panel B. No significant difference (left to right: $p=0.22, p=0.22, p=0.16$ ), as determined by two-sided t-test with 95\% confidence interval, in plant survival between BCC0191 WT and BCC0191 $\Delta \mathrm{c} 3$ at all inoculum levels. $n=10$ seeds per condition (seed coat) and dosage (cfu/seed), repeated in triplicate. Centre bar represents mean, and error bars represent standard error. 


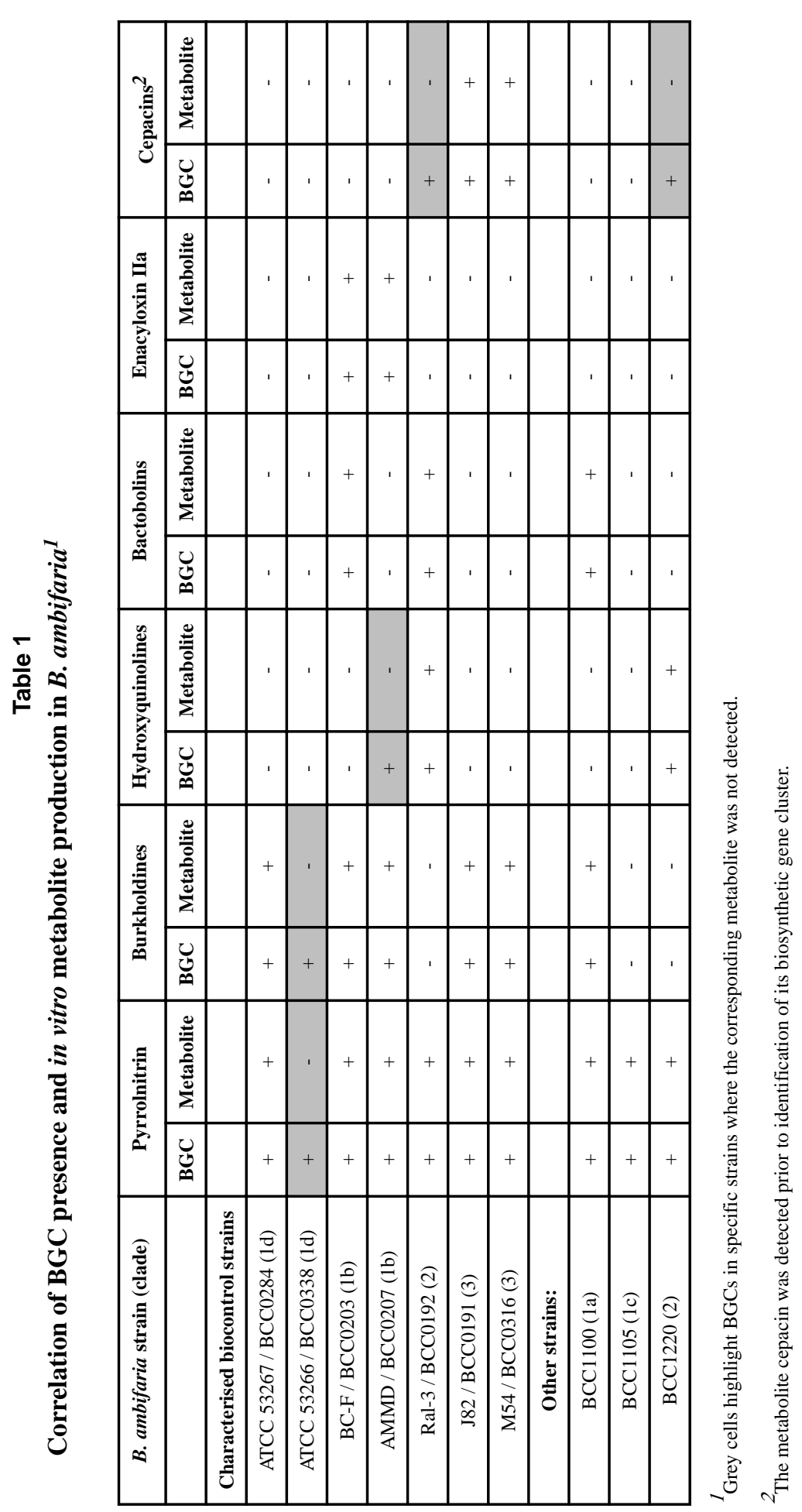

